\title{
LES TRÉMATODES DES GENRES MESOSTEPHANUS LUTZ, 1935 ET MESOSTEPHANOIDES DUBOIS, 1951 (STRIGEATA : CYATHOCOTYLOIDEA)
}

\author{
G. DUBOIS*
}

RÉSUMÉ. Cette étude complète notre travail de 1975. Les genres Mesostephanus et Mesostephanoides sont l'objet d'une révision et d'une définition. Neuf espèces sont redécrites. Une clé de détermination est proposée.

\section{The Trematodes of the genera Mesostephanus Lutz, 1935 and Mesostepha- noides Dubois, 1951 (Strigeata: Cyathocotyloidea)}

SUMMARY. This study finishes off our work of 1975. The genera Mesostephanus and Mesostephanoides are revised and defined. Nine species are redescribed. A key to species is proposed.

\section{Introduction}

Les espèces des genres Mesostephanus et Mesostephanoides ont en commun un corps indivis, terminé par un appendice dorso-subterminal dans lequel ne pénètrent jamais les gonades. Les follicules vitellogènes, confinés en arrière de l'acetabulum équatorial ou pré-équatorial, sont groupés en couronne souvent excentrée ou en fer à cheval autour de l'organe tribocytique. Les métacercaires se développent dans des Poissons.

Mesostephanus (syn. Gelanocotyle Sudarikov, 1961) possède un cirre petit, un sphincter vaginal, des gonades subsistant pendant la maturité sexuelle. Le rapport de la longueur du corps au diamètre de la couronne vitelline est compris entre 1,7 et 3,4. Parasites d'Oiseaux, plus rarement de Mammifères.

Espèce type : $M$. fajardensis (Price, 1934) Lutz, 1935.

Mesostephanoides possède un cirre relativement grand, pouvant atteindre, chez l'espèce type, 200 à $300 \mu \mathrm{m}$ de longueur et jusqu'à une trentaine de $\mu \mathrm{m}$ de diamètre à l'état de protrusion. Pas de sphincter vaginal. Les gonades disparaissent au cours de la maturité sexuelle. Le rapport de la longueur du corps au diamètre de la couronne vitelline est compris entre 3,5 et 4,5. Parasites de Reptiles (Serpents).

Espèce type : M. burmanicus (R. C. Chatterji, 1940) Dubois, 1951.

\footnotetext{
* Grand-Rue 12, CH-2035 Corcelles.

Accepté le 13 janvier 1982
} 
L'ontogénie complète n'est connue que pour quelques espèces du genre Mesostephanus : M. appendiculatoides (Price, 1934) [Hutton et Sogandares-Bernal 1960b], M. appendiculatus (Ciurea, 1916) [Martin 1961] et M. yedeae Dennis, 1968, syn. de appendiculatoides [Dennis et Penner 1971]. Le premier hôte intermédiaire est un Mollusque marin de la sous-classe des Prosobranches et de la superfamille des Cerithioidea Fleming : genres Cerithium Bruguière et Cerithidea Swainson'1.

Ce caractère biologique, à lui seul, suffirait déjà à écarter de la liste des Mesostephanus (cf. Dubois 1975b, pp. 447-448) M. kentuckiensis (Cable, 1935), dont les altrices se développent dans des Prosobranches d'eau douce (Mélaniidés : diverses espèces du genre Goniobasis, en particulier, genres Anoplocamus et Mudalia). Au surplus, le corps de ce Ver est bipartite, quoique faiblement bisegmenté, et le second segment contient les gonades (cf. Myer 1960, pl. II, fig. 8) ; les follicules vitellogènes sont accumulés dans l'organe tribocytique. C'est pourquoi Hoffman et Dunbar (1963) l'ont transféré dans le genre Neogogatea (Chandler et Rausch, 1947), transfert que nous avons approuvé (1975a, p. 39).

\section{Genre Mesostephanus Lutz}

\section{Mesostephanus appendiculatoides (Price, I934) Lutz, I935 (fig. I)}

Syn. Mesostephanus yedeae Dennis, 1968.

Le travail de Dennis et Penner (1971) permet de confirmer la mise en synonymie présumée (cf. Dubois 1975b, pp. 448 et 449) de Mesostephanus yedeae Dennis, 1968 avec $M$. appendiculatoides (Price, 1934). Les deux taxons, dont le cycle vital a été expérimenté en Floride (voir celui du second par Hutton et Sogandares-Bernal $1960 b$ ), utilisent Cerithium muscarum Say comme hôte intermédiaire des altrices.

Le caractère distinctif de l'espèce consiste dans la petitesse de l'acetabulum, dont le diamètre ne mesure que 28-32/20-32 $\mu$ m (d'après Price 1934), 26-42/34-42 $\mu \mathrm{m}$ (d'après Caballero, Grocott et Zerecero 1953), 30-35/23-30 $\mu \mathrm{m}$ (d'après Dennis et Penner 1971) ${ }^{2}$.

Distribution géographique : République Dominicaine, Porto Rico, Floride, République de Panama.

Hôte type : Pelecanus occidentalis L.

I. Les cercaires de Mesostephanus appartiennent au sous-groupe Vivax Sewell, I922 (emend. Dubois, 195I) et à sa subdivision a). Elles sont caractérisées par de longs fourchons avec replis natatoires sur toute leur longueur, par une ébauche acétabulaire, une ventouse buccale ressemblant à un organe céphalique protrusible et traversé par les conduits des glandes de pénétration. Système excréteur de formule $2[(3+3+3)+(3+3+(3)]=36$ protonéphridies. Les sporocystes sont annelés, leurs parois étant cerclées de bandes contractiles transversales qui leur donnent l'apparence de petits Chaetopodes. Ils se développent chez Cerithium muscarum Say. (pour $M$. appendiculatoides), Cerithium atratum (Born.) (pour $M$. fajardensis), Cerithidea californica Haldeman (pour $M$. appendiculatus, aux Etats-Unis).

2. L'acetabulum du Mesostephanus appendiculatoides déposé par Robert F. Hutton à 1'U. S. Nat. Mus., Helm. Coll. no $5624 \mathrm{I}$, mesure $32 \mu \mathrm{m}$ de diamètre transversal. Celui du paratype de $M$. yedeae (ibid., no 7 I040) ne dépasse pas $30 \mu \mathrm{m}$ dans le même sens. 
Iconographie : Price 1934, pl. I, fig. 7 ; Dubois 1938, fig. 326 (paratype) ; Caballero, Grocott et Zerecero 1953, fig. 3 ; Hutton et Sogandares-Bernal 1960a, fig. 1 C ; Dennis et Penner 1971, fig. 5 et 6 .

Mesostephanus appendiculatus (Ciurea, I9I6) Lutz, I935 (fig. 2)

Syn. Prohemistomum appendiculatum Ciurea, 1916 ;

Mesostephanus longisaccus Chandler, 1950 ;

Mesostephanus skworzowi Petrov, 1950 ;

Mesostephanus sp. Malczewski, 1962;

Mesostephanus alopicis Malczewski, 1964.

Décrite par Ciurea, en 1916, comme parasite de l'intestin grêle de trois chiens et d'un chat, en Roumanie, l'espèce a été retrouvée par Price, en 1928, aux ÉtatsUnis, chez un chien, puis par Prendel, en 1930, en Ukraine, chez le même hôte. En 1950, Chandler, au Texas, puis Petrov, en URSS, la redécrivent sous les noms de M. longisaccus ${ }^{1}$ et de $M$. skworzowi, comme parasites de Canis familiaris L. et de Felis catus L., respectivement. Puis Malczewski, en 1962 et 1964, la signale en Pologne sous les noms de Mesostephanus sp. et M. alopicis, chez le Renard polaire (Alopex lagopus L.). Enfin, Martin (1961) en décrit le cycle vital à partir de la cercaire issue de Cerithidea californica Haldeman, en Californie.

Deux des caractéristiques de l'espèce consistent dans la grandeur du pharynx (61-84/50-75 $\mu \mathrm{m})$ qui, parfois, peut surpasser celle de la ventouse buccale, et dans la structure du court œsophage (25-45/20-25 $\mu \mathrm{m})$, dont les parois musculeuses sont constituées de fibres circulaires bien visibles, qui délimitent une lumière étroite (caractères confirmés par les observations de Martin (1961, p. 279) : ventouse buccale 47-78/40-59 $\mu \mathrm{m}$; pharynx 37-58/31-44 $\mu \mathrm{m}$; " esophagus approximately one-half pharyngeal length, with transverse muscle fibers ").

Distribution géographique : Europe, Chine, États-Unis.

Hôtes types: Canis familiaris L. et Felis catus L. (expérim.).

Iconographie : Ciurea 1916, fig. 1-2; Tubangui 1922, pl. IV, fig. 6 (d’après Ciurea) ; Prendel 1930, fig. 1 (orig.) ; Dubois 1938, fig. 327 (orig.).

Mesostephanus fajardensis (Price, I934) Lutz, I935 ( fig. 3)

Syn. Prohemistomum fajardensis Price, 1934.

Décrite par Emmett W. Price, en 1934, comme parasite de l'intestin d'une Sula leucogaster (Bodd.) capturée à l'est de Porto Rico (entre l'Isla Palominos et Fajardo), cette espèce type se distingue de ses congénères par la petitesse et le grand nombre de ses œufs à coque relativement épaisse. Elle a été retrouvée par Lutz (1935) au

I. Les exemplaires de $M$. longisaccus sont jeunes bien qu'ovigères. Ils ne mesurent que 0,75 à $1,05 \mathrm{~mm}$ de longueur. Leur pharynx a $63-80 \mu \mathrm{m}$ de long et $50-75 \mu \mathrm{m}$ de diamètre. La poche du cirre, d'un diamètre de 80 à $90 \mu \mathrm{m}$, s'allonge jusqu'à mi-longueur du corps ou même au-delà . De même, $M$. alopicis ne mesure que $0,52-0,90 \mathrm{~mm}$ de longueur, mais le pharynx est relativement grand $(33-52 / 37-72 \mu \mathrm{m})$. La poche du cirre, qui s'étire, d'un diamètre de $30-72 \mu \mathrm{m}$, atteint le niveau de l'organe tribocytique. 


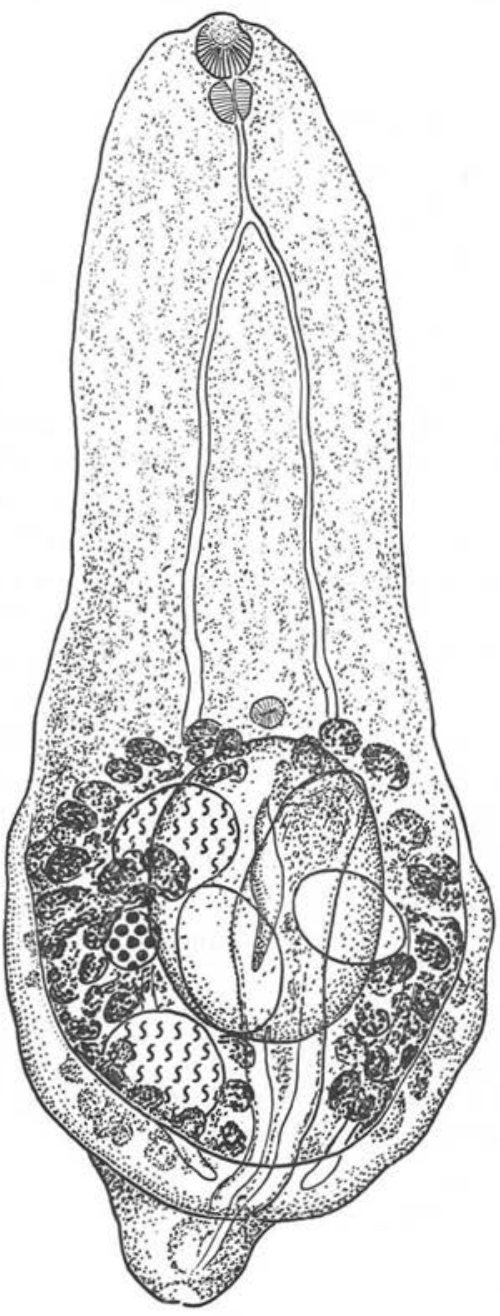

FIG. I

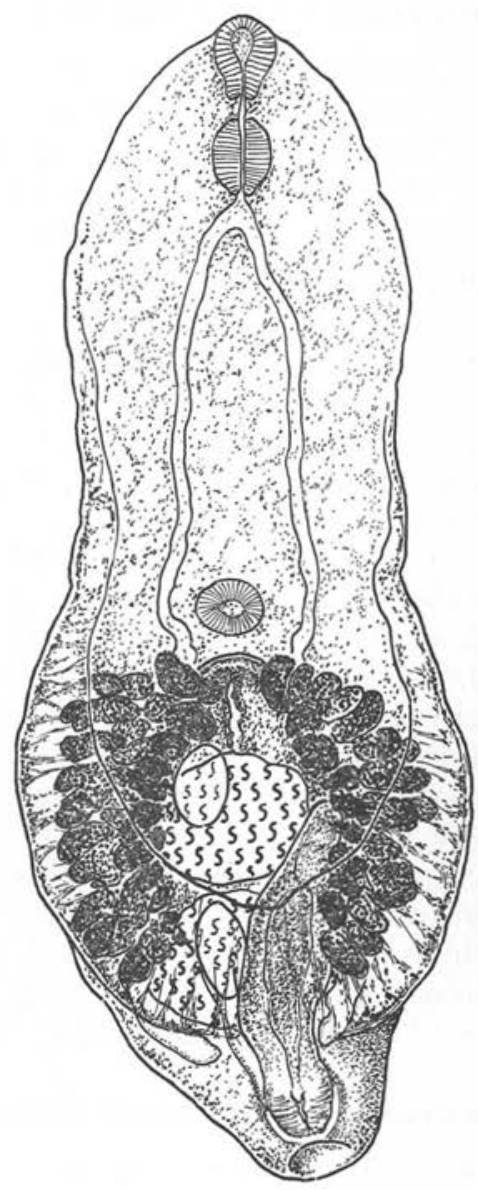

FIG. 2

FIG I. - Mesostephanus appendiculatoides (Price), de Pelecanus occidentalis L. Longueur o,82 mm. Vue ventrale. Paratype, U.S.N.M. Helm. Coll., no 8702.

FIg. 2. - Mesostephanus appendiculatus (Ciurea), de Canis familiaris L. Longueur 1,35 mm. Vue ventrale. Exemplaire de la collection Ciurea. 
Brésil, chez Sula brasiliensis Spix, puis par Cable, Connor et Balling (1960) chez l'hôte type, à Lajas (Parguera), Porto Rico, qui la redécrivirent. L'espèce fut encore signalée par Dubois (1958, p. 76) qui en trouva deux grands exemplaires dans la collection recueillie par June Mahon, en Californie, chez Sula sula (L.).

De l'ensemble des descriptions, on retiendra les données suivantes : longueur du corps 0,86-1,85 $\mathrm{mm}$; largeur 0,38-0,80 $\mathrm{mm}$; appendice dorso-postérieur 0,08 $0,18 / 0,14-0,35 \mathrm{~mm}$. Ventouse buccale 44-72/53-77 $\mu \mathrm{m}$; acetabulum 45-90/62-125 $\mu \mathrm{m}$; organe tribocytique $300-400 / 300-360 \mu \mathrm{m}$; pharynx 32-59/18-36 $\mu \mathrm{m}$; œsophage $29-$ $145 \mu \mathrm{m}$. Ovaire 75-143/70-161 $\mu \mathrm{m}$; testicule antérieur 75-215/77-170 $\mu \mathrm{m}$; testicule postérieur 98-286/79-197 $\mu \mathrm{m}$; poche du cirre 322-609/80-150 $\mu \mathrm{m}$; sphincter vaginal 80-147/93-166 $\mu \mathrm{m}$. Eufs 44-64/32-43 $\mu \mathrm{m}$, au nombre de 16 à 60 dans l'utérus.

Distribution géographique : Porto Rico, Brésil, Californie.

Hôte type : Sula leucogaster (Bodd.).

Iconographie : Price 1934, pl. I, fig. 6 ; Dubois 1938, fig. 328 (paratype) ; Cable, Connor et Balling 1960, pl. I, fig. 3 (orig.).

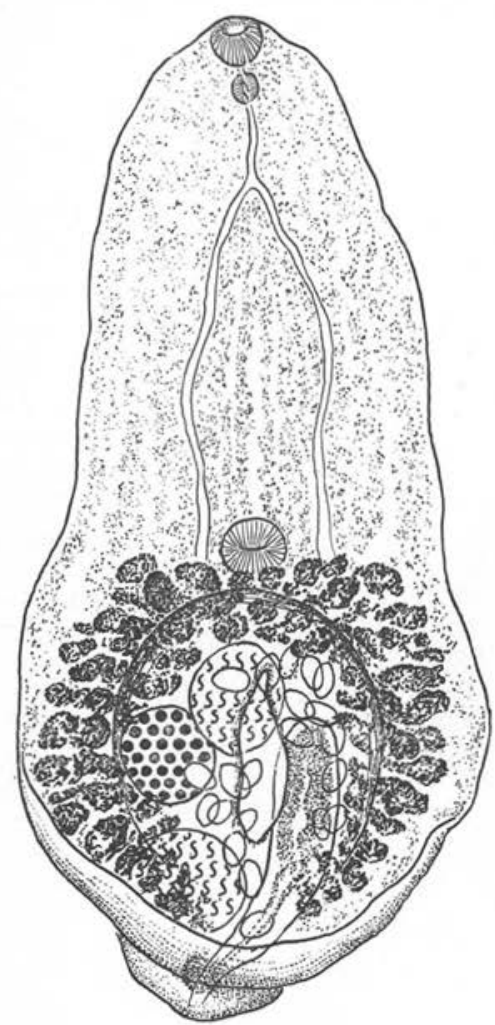

FIG. 3. - Mesostephanus fajardensis (Price), de Sula leucogaster (Bodd.). Longueur I,I5 mm. Vue ventrale. Paratype, U.S.N.M. Helm. Coll., no 8697. 
Mesostephanus milvi Yamaguti, I939 (fig. 4)

Syn. Mesostephanus indicus Vidyarthi, 1948 nec Mehra, 1947;

Mesostephanus sp. Richard, 1964 ;

Mesostephanus cordiformis Oshmarin, Mamaev et Lebedev, 1970 ;

Mesostephanus sp. Oshmarin, Mamaev et Lebedev, 1970.

Décrite par Yamaguti (1939), au Japon, comme parasite de Milvus milvus lineatus Gray, cette espèce a été retrouvée plusieurs fois en Égypte chez Milvus migrans (Bodd.), mais aussi dans des chats domestiques et le chien (cf. Dubois et Pearson 1963, pp. 85-89; Dubois $1969 a$, p. 20 ; 1978, p. 614). Elle s'identifie au Mesostephanus indicus Vidyarthi, 1948 nec Mehra, 1947, de Milvus migrans, des environs de Lucknow (cf. Vidyarthi 1948, p. 26, Dubois et Pearson 1963, pp. 86, 89). Mme Josette Richard (1964, pp. 517-519) apparente à milvi son Mesostephanus sp. trouvé à Madagascar chez un canard sauvage. Enfin, Oshmarin, Mamaev et Lebedev (1970, pp. 115-118) décrivent un Mesostephanus cordiformis et un Mesostephanus sp., tous deux de Milvus migrans (syn. M. korschun), provenant de Haïphong (Nord Viêtnam) et qui présentent toutes les caractéristiques de $M$. milvi (cf. Dubois 1977, p. 42$)^{1}$.

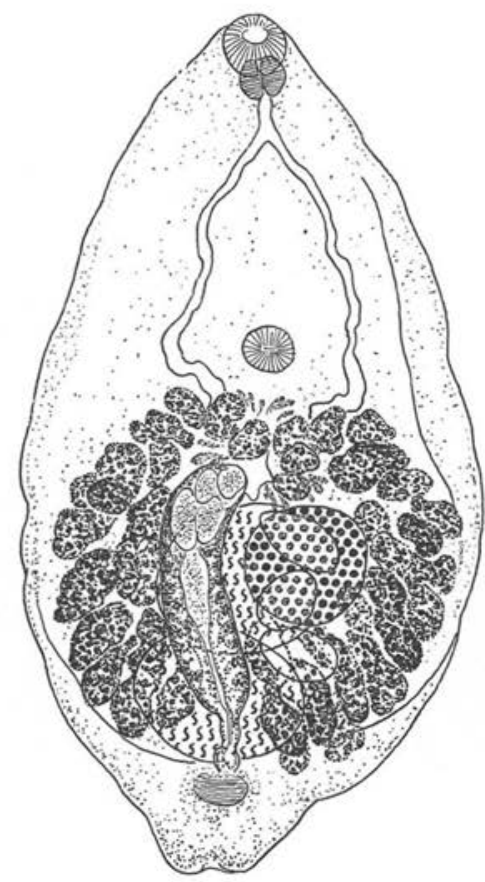

Fig. 4. - Mesostephanus milvi Yamaguti, de Milvus migrans (Bodd.).

Longueur I, I3 mm. Vue ventrale. Collection William $\mathrm{H}$. Wells, $\mathrm{n}^{0} \mathrm{PE}-4004-\mathrm{T}$.

I. Yamaguti (1939), Vidyarthi (1948) et Oshma1in et al. (I970) n'ont pas observé de sphincter vaginal. Nous avons relevé (Dubois et Pearson op. cit., pp. 86, 89) le fait que ce muscle n'apparaît qu'à la faveur de fixations et colorations appropriées (notamment au carmin aluné). 
Corps ovale, cochléariforme, long de 0,90 à $1,65 \mathrm{~mm}$. Ventouse buccale plus grande que l'acetabulum faiblement musculeux et situé aux 2/5 de la longueur du corps. Pharynx plus petit que la ventouse buccale, mais plus musculeux. Esophage court. Vitellogènes recouvrant complètement les gonades. Partie distale (post-prostatique) de la poche du cirre équivalant au $1 / 5$, au $1 / 6$ ou au $1 / 7$ de la longueur de l'organe.

Distribution géographique : Japon, Nord Viêtnam, Inde, Égypte, Madagascar. Hôte type : Milvus migrans lineatus Gray.

Iconographie : Yamaguti 1939, pl. XXVI, fig. 56 ; Vidyarthi 1948, fig. 2; Dubois et Pearson 1963, fig. 7-10; Richard 1964, fig. 6; Oshmarin, Mamaev et Lebedev 1970, fig. 96 et 97.

\section{Mesostephanus neophocae Dubois et Angel, I976 ( fig. 5)}

Vingt-sept spécimens de ce Prohémistomidé ont été récoltés dans l'instestin d'un Lion de mer, Neophoca cinerea (Péron et Lesueur), rejeté sur un rivage près d'Adelaïde (sud de l'Australie), le 29 janvier 1975. La localité serait "St Vincent Gulf, S. Austr. ". S'agit-il d'une infestation normale, car les Otaries se nourrissent aussi d'oiseaux, y compris les Cormorans dont quelques-uns peuvent héberger des Mesostephanus?

Un jeune exemplaire de cette espèce avait été récolté en février 1941 par le Dr John C. Pearson dans un Éléphant de mer, Mirounga leonina (L.), captif dans le Glenelg Aquarium, S. Aust. Il présentait tous les caractères spécifiques et contenait un seul œuf $(120 / 78 \mu \mathrm{m})$.

$M$. neophocae peut être caractérisé ainsi : longueur du corps 1,20-1,45 mm, largeur 0,50-0,60 mm. Ventouse buccale 85-90/90-99 $\mu \mathrm{m}$; acetabulum 70-89/85$94 \mu \mathrm{m}$, situé aux 33-43/100 ; pharynx 57-70/55-65 $\mu \mathrm{m}$; œsophage 30-60 $\mu \mathrm{m}$; organe tribocytique généralement rétracté, arrondi en protrusion. Ovaire 85-100/95-115 $\mu \mathrm{m}$; testicule antérieur 170-220/150-190 $\mu \mathrm{m}$; testicule postérieur 190-220/150-185 $\mu \mathrm{m}$; poche du cirre 415-500/80-100 $\mu \mathrm{m}$, s'étendant jusqu'au niveau intertesticulaire ou au tiers postérieur du premier testicule, où se situe l'ovaire ; sphincter vaginal 35-40/ 42-50 $\mu \mathrm{m}$. Vitellogènes s'étendant du front de l'organe tribocytique jusqu'à la seconde moitié, voire le pôle caudal du deuxième testicule; rapport de la longueur $\mathrm{du}$ corps au diamètre moyen de la couronne vitelline $=2,4-2,8$. Eufs 105-120/ 75-85 $\mu \mathrm{m}$.

Distribution géographique : Australie méridionale.

Hôte type : Neophoca cinerea (Péron et Lesueur).

Iconographie: Dubois et Angel 1976, fig. 1.

Mesostephanus odhneri (Travassos, I924) Lutz, I935 (fig. 6)

Syn. Prohemistomum odhneri Travassos, 1924 nec Mehra, 1947 ;

Mesostephanus infecundus Lutz, 1935 ;

Mesostephanus appendiculatoides Cable, Connor et Balling, 1960 nec Price, 1934 (vide Dubois 1975, pp. 449 et 456). 
La description originale était basée sur l'examen de nombreux spécimens obtenus expérimentalement dans l'intestin de quelques Nyctanassa violacea (L.), par infestation au moyen de kystes trouvés dans le tissu sous-cutané d'un Perciforme du genre Haemulon, au Brésil.

La redescription suivante est basée sur l'examen des nombreuses préparations de la Collection A. Lutz, déposées à l'Institut Oswaldo Cruz de Rio de Janeiro, et

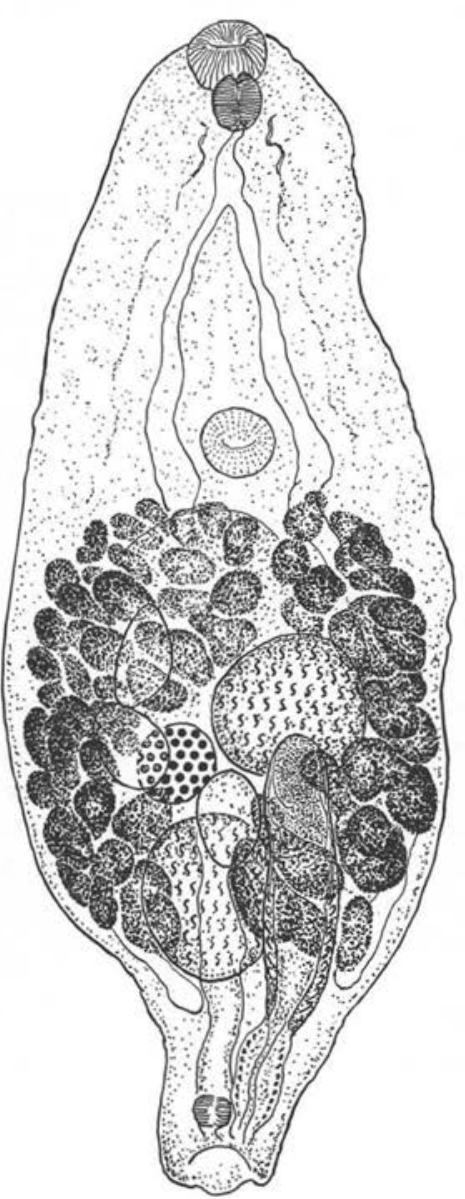

FIG. 5

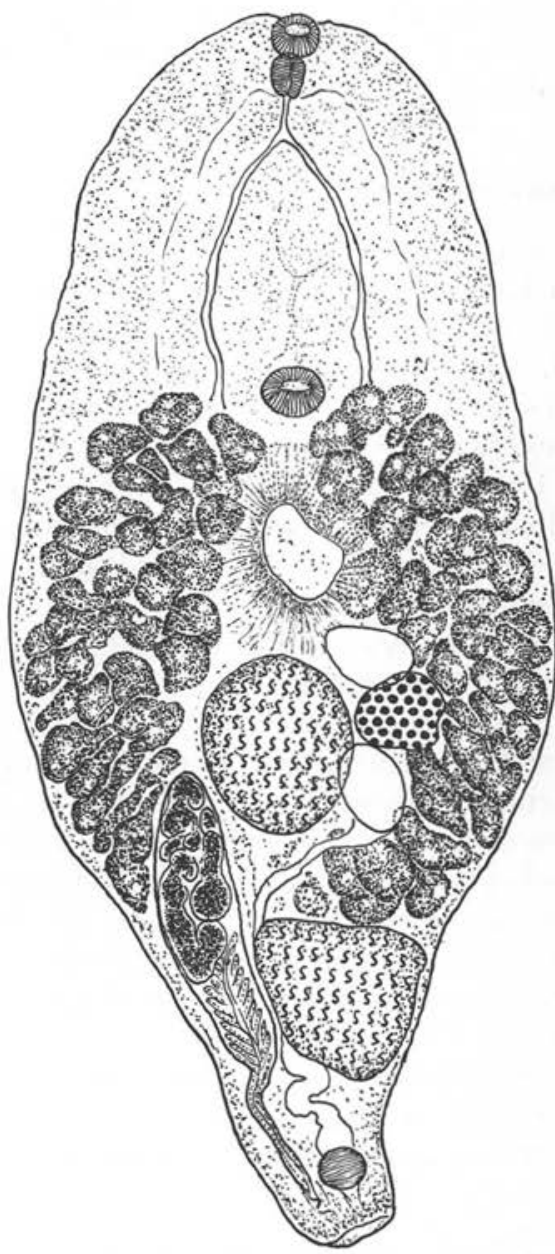

FIG. 6

Fig. 5. - Mesostephanus neophocae Dubois et Angel, de Neophoca cinerea (Péron et Lesueur). Longueur I,34 mm. Vue ventrale. Holotype, South Austr. Mus., no V 75.

Fig. 6. - Mesostephanus odhneri (Travassos), de Fregata aquila (L.).

Longueur I,59 mm. Vue ventrale. Collection A. Lutz, $\mathrm{n}^{0} 2543^{8}$. 
provenant soit de Sula sula (L.) [= S. brasiliensis Spix], soit de Fregata aquila (L.). Cf. Dubois 1970, pp. 192-193.

Corps linguiforme : 0,80-1,34/0,40-0,59 mm (chez Sula), 1,12-2,23/0,57-0,90 mm (chez Fregata). Ventouse buccale 43-63/38-57 $\mu \mathrm{m}$; pharynx 32-63/22-45 $\mu \mathrm{m}$; œsophage 30-90 $\mu \mathrm{m}$; acetabulum 45-66/52-84 $\mu \mathrm{m}$, situé aux 30-45/100 de la longueur du Ver; organe tribocytique 180-530/130-320 $\mu \mathrm{m}$. Ovaire 75-125/65-95 $\mu \mathrm{m}$, au niveau du testicule antérieur ; testicules 170-300/130-275 $\mu \mathrm{m}$; vitellogènes s'étendant du bord postérieur de l'acetabulum au niveau intertesticulaire et constituant une couronne dont le rapport du diamètre moyen à la longueur du corps égale 2,06 à 3 ; sphincter vaginal $30-42 / 35-50 \mu \mathrm{m}$; longueur de la poche du cirre $500-830 \mu \mathrm{m}$; diamètre du cirre 10-16 $\mu \mathrm{m}$. Eufs 100-115/66-73 $\mu \mathrm{m}$, au nombre de 1 à 50 (chez Sula), 1 à 28 (chez Fregata).

Distribution géographique : Brésil, Porto Rico.

Hôte type expérimental : Nyctanassa violacea (L.).

Iconographie : Travassos 1924, fig. 1-3 ; Lutz 1935, pl. I, fig. 8 ; Cable, Connor et Balling 1960, pl. II, fig. 5 (faussement attribuée à $M$. appendiculatoides); Dubois 1970, fig. 1 et 2 .

\section{Species inquirenda}

\section{Mesostephanus parappendiculatus Baer, I959}

J. G. Baer n'a trouvé qu'un seul exemplaire adulte (anormalement élargi dans la région antérieure après fixation) parmi plusieurs formes larvaires, dans l'intestin d'un Pelecanus rufescens Gm. capturé, en 1958, à l'embouchure de la Talya dans le lac Édouard (Congo-Kinshasa = Zaïre). Ce Ver est sommairement décrit (1959, p. 11, fig. 2) : longueur $0,91 \mathrm{~mm}$, largeur $0,64 \mathrm{~mm}$; ventouse buccale $64 \mu \mathrm{m}$ de diamètre ; acetabulum $79 \mu \mathrm{m}$; pharynx $45 / 34 \mu \mathrm{m}$; organe tribocytique $160 / 114 \mu \mathrm{m}$; ovaire $57 \mu \mathrm{m}$; testicule postérieur $128 \mu \mathrm{m}$; poche du cirre $204 / 45 \mu \mathrm{m}$. Un seul œuf mesurant $91 / 50 \mu \mathrm{m}$.

A la suite de ces espèces, il faut en citer quatre autres dont on trouvera la description dans notre publication de $1975 b$ :

M. cubaensis Alegret, 1941, parasite de Phalacrocorax auritus floridanus (Audub.), dans l'île de Cuba, et dont Dubois (1951, p. 652, note 2 et fig. 3) confirme l'attribution générique par l'observation du sphincter vaginal sur le matériel original ;

$M$. dottrensi Baer, 1957, trouvé dans l'intestin d'une chouette pêcheuse, Scotopelia peli Bonap., en Côte-d'Ivoire, dont le matériel type nous a permis de compléter les données originales ;

M. haliasturis Tubangui et Masiluñgan, 1941, hébergé par Haliastur indus intermedius Blyth, aux Philippines, et redécrit par Dubois et Angel (1972) d'après un matériel recueilli en Australie, chez Pelecanus conspicillatus Temm. qui paraît être un des hôtes normaux, cité aussi par Dubois et Pearson (1967) ; 
M. microbursa Caballero, Grocott et Zerecero, 1953, parasite de Pelecanus occidentalis californicus Ridway, en République de Panama et dans l'île de Coronado, en Basse Californie, redécrit par Dubois (1958) d'après un matériel récolté par June Mahon dans le même hôte et la même île.

\section{Genre Mesostephanoides Dubois}

Mesostephanoides burmanicus (R.C. Chatterji, I940) Dubois, I95I ( fig. 7a, b)

Syn. Mesostephanus burmanicus Chatterji, 1940 ;

Gogatea burmanicus (Chatterji, 1940) H. R. Mehra, 1943.

Cette espèce, décrite par R. C. Chatterji (1940, pp. 395-398, fig. 4 et 5) comme parasite intestinal d'un Colubridé, Enhydris enhydris (Schneider) provenant de Rangoon (Birmanie), a été transférée dans le genre Gogatea Lutz, 1935 par H. R. Mehra (1943, 1947), puis choisie comme type de Mesostephanoides par Dubois (1951, pp. 659, $660)$.

La description suivante est basée sur les travaux des deux auteurs indiens : longueur du Ver 0,85-1,60 mm ; partie antérieure 0,63-1,00/0,25-0,46 mm ; appendice dorso-subterminal $0,12-0,25 / 0,09-0,17 \mathrm{~mm}$. Ventouse buccale $58-73 / 75-83 \mu \mathrm{m}$; acetabulum $30-38 / 33-45 \mu \mathrm{m}$; organe tribocytique $135-240 / 120-220 \mu \mathrm{m}$; pharynx $34-$ 52/30-37 $\mu \mathrm{m}$; œsophage 87-182 $\mu \mathrm{m}$. Ovaire 50-60 $\mu \mathrm{m}$; testicule antérieur 90-95/ $40-60 \mu \mathrm{m}$; testicule postérieur 90-95/70-90 $\mu \mathrm{m}$ (les gonades disparaissent dès la maturité sexuelle et le développement complet des œufs) ; poche du cirre 240-540/ 50-80 $\mu \mathrm{m}$; cirre 228-285/24-33 $\mu \mathrm{m}$ en protrusion; metraterm 120-150/21-29 $\mu \mathrm{m}$. Eufs 98-140/72-96 $\mu \mathrm{m}$, au nombre de 1 à 3 dans l'utérus.

Distribution géographique : Birmanie (Rangoon).

Hôte type : Enhydris enhydris (Schneider).

Iconographie : R. C. Chatterji 1940, fig. 4 et 5 ; H. R. Mehra 1947, fig. 3 ; Dubois 1951, fig. 6 et 7 (d'après ces deux auteurs).

\section{Mesostephanoides taiwanensis Fischthal et Kuntz, I975 (fig. 8)}

Ce Cyathocotylide a été découvert dans l'intestin grêle d'un Colubridé, Enhydris chinensis (Gray), à Taiwan (Préfecture de Taipei), le 19 juin 1962. La description est basée sur l'examen de l'holotype (U. S. Nat. Mus., Helm. Coll. no 73148).

Corps long de $1,22 \mathrm{~mm}$, à partie antérieure ovale, $0,87 / 0,45 \mathrm{~mm}$, finement spinuleuse, prolongée par un appendice dorso-subterminal mesurant 0,35/0,19 mm. Ventouse buccale $100 / 119 \mu \mathrm{m}$; acetabulum presque aussi grand, $85 / 97 \mu \mathrm{m}$; organe tribocytique lobulé, $340 / 250 \mu \mathrm{m}$, à ouverture médiane longue de $200 \mu \mathrm{m}$, tréflée en avant, bordée d'une cuticule épaisse et striée; pharynx $85 / 58 \mu \mathrm{m}$; œsophage? $73 \mu \mathrm{m}^{1}$. Ovaire $95 / 90 \mu \mathrm{m}$; testicule antérieur $125 / 85 \mu \mathrm{m}$; testicule postérieur $135 /$

I. La mesure donnée dans la description originale est sujette à caution en raison du repli de la partie céphalique de l'holotype. 


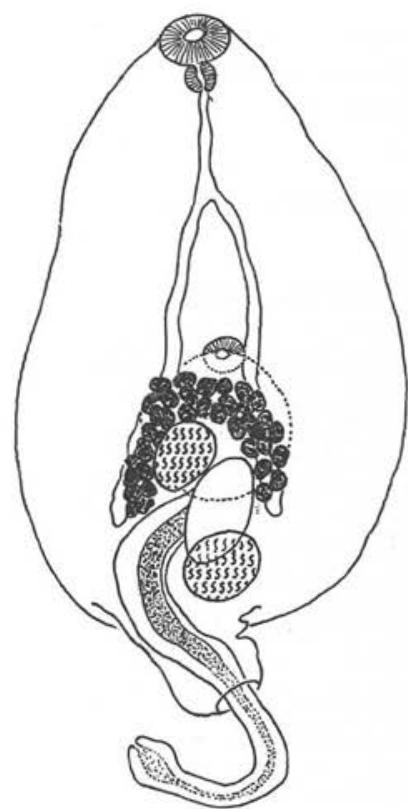

FIG. 7 a

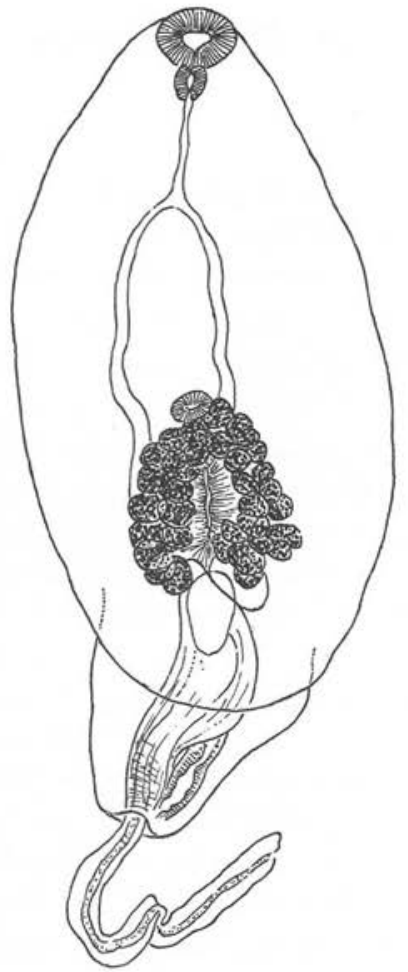

FIG. 7 b

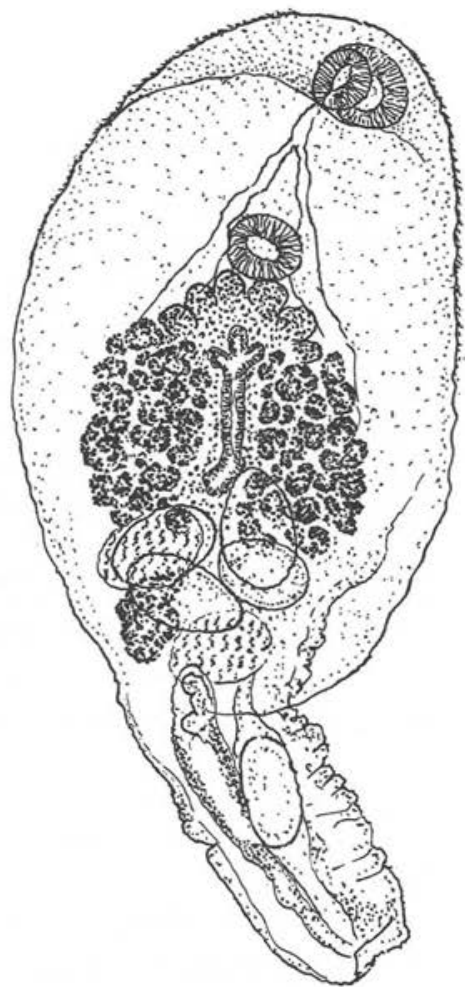

FIG. 8

FIG. 7. - Mesostephanoides burmanicus (R. C. Chatterji), de Enhydris enhydris (Schneider).

a) d'après Chatterji, fig. 4. Vue dorsale.; b) d'après Mehra, fig. 3. Vue ventrale.

FIG. 8. - Mesostephanoides taiwanensis Fischthal et Kuntz, de Enhydris chinensis (Gray). Longueur I,22 mm. Vue ventrale. Holotype, U.S.N.M. Helm. Coll., no 73148 .

$80 \mu \mathrm{m}$; vitellogènes disposés en deux champs latéraux semi-elliptiques, dorsaux par rapport à l'organe tribocytique ; poche du cirre $400 / 53 \mu \mathrm{m}$, s'allongeant jusqu'au niveau du bord caudal du second testicule ; cirre spinuleux, 190/22 $\mu \mathrm{m}$ (en rétraction $)^{1}$; metraterm $340 \mu \mathrm{m}$ de long. (Eufs 131-144/84-100 $\mu \mathrm{m}^{2}$, au nombre de 5 dans l'utérus.

Distribution géographique : Taiwan (Taipei Prefecture).

Hôte type : Enhydris chinensis (Gray).

Iconographie : Fischthal et Kuntz 1975, fig. 15.

I. La description originale indique $68 / 22 \mu \mathrm{m}$.

2. D'après nos mesures sur les œufs non déformés. (L'œuf engagé dans le metraterm est notablement allongé.) 


\section{Clé de détermination}

\section{Genre Mesostephanus Lutz}

1. Eufs petits (44-64/31-43 $\mu \mathrm{m}$ ), nombreux (jusqu'à 60), à coque relativement épaisse. Parasite de Sula. Porto Rico, Brésil. ... M. M. fajardensis (Price, 1934)1.

- Eufs gros $(80-125 / 50-92 \mu \mathrm{m}$ ), rares ou peu nombreux (jusqu'à 28). ...... 2

2. Vitellogènes à limite postérieure intertesticulaire. $\ldots \ldots \ldots \ldots \ldots \ldots \ldots, 3$

- Vitellogènes outrepassant le niveau intertesticulaire pour recouvrir partiellement ou entièrement le second testicule. .................... 4

3. Corps petit, $0,91 \mathrm{~mm}$. Pharynx plus petit que la ventouse buccale. Parasite de Pelecanus rufescens Gm. Congo Kinshasa (Lac Edouard). ............

M. parappendiculatoides Baer, 1959.

- Corps moyen, 0,80-2,23 mm. Pharynx de même longueur que la ventouse buccale, mais plus musculeux. Parasite de Sula et de Fregata. Brésil. ......

$$
\text { M. odhneri (Travassos, 1924) } 2 \text {. }
$$

4. Pharynx (61-84/50-75 $\mu \mathrm{m})$, presque aussi grand, parfois même plus gros que la ventouse buccale. (Esophage court (25-45/20-25 $\mu \mathrm{m})$, musculeux, constitué par un manchon de fibres circulaires bien visibles, qui délimitent une lumière étroite. Roumanie, Pologne, URSS, États-Unis. .............................. M. appendiculatus (Ciurea, 1916).

- Pharynx plus petit que la ventouse buccale. Esophage peu musculeux. ... 5

5. Acetabulum faiblement musculeux, très petit $(26-42 / 23-42 \mu \mathrm{m})$. République dominicaine, Porto Rico, Floride, Panama. ................ .............................. M. appendiculatoides (Price, 1934).

- Acetabulum de dimensions moyennes $(30-89 / 41-94 \mu \mathrm{m})$. . . . . . . . . . . 6

6. Ventouse buccale plus grande que l'acetabulum. ................ 7

- Ventouse buccale plus petite que l'acetabulum. ................. 9

7. Corps typiquement spathacé, de petite taille $(<1 \mathrm{~mm})$. Côte-d'Ivoire, Égypte. .............................. M. dottrensi Baer, 1957.

— Corps ovale à lancéolé (généralement $>1 \mathrm{~mm}$ ). .............. 8

8. Partie distale (post-prostatique) de la poche du cirre ne représentant que le $1 / 5$, le $1 / 6$ ou le $1 / 7$ de la longueur de l'organe. Japon, Viêtnam, Inde, Égypte, Zaïre, Madagascar. ................. M. milvi Yamaguti, 1939.

- Partie distale (post-prostatique) de la poche du cirre équivalant au $1 / 3$ de la longueur de l'organe. Australie. ........ neophocae Dubois et Angel, 1976.

I. Cette espèce aurait été observée par Lutz (1935), au Brésil, qui la cite sans la décrire, sous le nom de $M$. prolificus.

2. Cette espèce aurait été observée par Lutz (1935), au Brésil, qui la cite sans la décrire, sous le nom de $M$. infecundus. Cable, Connor et Balling (1960, pp. 197-198, fig. 5) l'ont retrouvée et décrite sous le nom de Mesostephanus appendiculatoides (Price, 1934) Lutz, 1935. 
9. Corps de petite taille $(0,62$ à $1,5 \mathrm{~mm}$ de long), à " épaules " bien marquées, d'où les bords latéraux se replient ventralement pour se réunir au-devant de l'appendice dorso-subterminal courtement tronconique. Philippines, Australie. ............. Maliasturis Tubangui et Masiluñgan, 1941.

- Corps plus grand $(1,2$ à $2 \mathrm{~mm})$, longuement elliptique à lancéolé. . . . . . 10

10. Appendice dorso-subterminal très réduit. Acetabulum aux 45-53/100 de la longueur du corps. Rapport de celle-ci au diamètre moyen de la couronne vitelline $=3,5-3,9$. Eufs $94-110 / 68-76 \mu \mathrm{m}$. Cuba. . . . . . . . . . . . . . M. cubaensis Alegret, 1941. Appendice dorso-subterminal relativement grand, long de 0,36 - à $0,55 \mathrm{~mm}$. Acetabulum situé aux 32-42/100 de la longueur du corps ${ }^{1}$. Rapport de celle-ci au diamètre moyen de la couronne vitelline $=2,5-3,2$. Eufs 115-

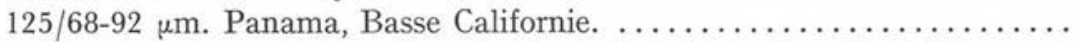
M. microbursa Caballero, Grocott et Zerecero, 1953.

Genre Mesostephanoides Dubois

Appendice dorso-subterminal 120-250/90-170 $\mu \mathrm{m}$. Acetabulum (30-38/33-45 $\mu \mathrm{m})$ 2 fois plus petit que la ventouse buccale. Pharynx 34-52/30-37 $\mu \mathrm{m}$. Vitellogènes disposés en fer à cheval. Longueur du metraterm 120-150 $\mu \mathrm{m}$. Birmanie.

M. burmanicus (R. C. Chatterji, 1940).

Appendice dorso-subterminal 355/187 $\mu \mathrm{m}$. Acetabulum $(85 / 97 \mu \mathrm{m})$ presque aussi grand que la ventouse buccale. Pharynx $85 / 58 \mu \mathrm{m}$. Vitellogènes disposés en deux champs latéraux semi-elliptiques. Longueur du metraterm $340 \mu \mathrm{m}$. Formose.

.................... M. taiwensis Fischthal et Kuntz, 1975.

Remerciements. Nous remercions le Dr Ralph Lichtenfels, de l'U. S. National Museum, à Beltsville Md., du prêt de l'holotype de Mesostephanoides taiwanensis Fischthal et Kuntz, d'un paratype de Mesostephanus yedeae Dennis, et d'une préparation de $M$. appendiculatoides (Price) déposée par Robert F. Hutton. Nous sommes gré au Dr B. Hörning, de l'Institut für Tierpa thologie, à Berne, de nous avoir procuré une photocopie du travail publié par l'Académie des Sciences de l'URSS (1970), dans lequel sont décrits les Mesostephanus cordiformis et Mesostephanus sp. de Oshmarin, Mamaev et Lebedev.

I. D'après Caballero, Grocott et Zerecero (1953, p. 398), l'acetabulum est plus grand (65$76 / 68-80 \mu \mathrm{m})$ que la ventouse buccale $(57 / 65-68 \mu \mathrm{m})$, tandis que la figure 5 le représente plus petit. Or, le premier de ces auteurs (in litt., 28 mars 1956) rectifiait la proportion en avouant que l'acetabulum " está mal dibujado ». 


\section{BIBLIOGRAPHIE}

Alegret M. J.: Contribución al conocimiento de los vermes parásitos de Phalacrocorax Auritus Floridanus, La Habana (Tesis), I94I, I-32.

BAER J. G. : Trématodes et Cestodes récoltés en Côte d'Ivoire, avec remarques sur la famille des Dicrocoeliidae Odhner et sur les parasites des Damans. Rev. suisse Zool., 1957, 64, 547-575.

BAer J. G. : Helminthes parasites. In : Explor. Parcs nat. Congo Belge, Mission Baer et Gerber, I959, I, I-80, Bruxelles.

Caballero E., Grocotr R. G., Zerecero C. : Helmintos de la Republica de Panama. IX. Algunos trematodos de aves marinas del Oceano Pacifico del Norte. An. Inst. Biol. Méx., 1953, 24, 39I-4I 4 .

Cable R. M., Connor R. S., Balling J. W. : Digenetic trematodes of Puerto Rican shore birds. Sci. Surv. P. R., 1960, 17, 19I-255.

Chander A. C. : Mesostephanus longisaccus, a new cyathocotylid trematode from a dog. J. Parasitol, 1950, 36, 90 .

Chatterji R. C.: Helminth parasites of the snakes of Burma, I. Trematoda. Philipp. J. Sci., I940, $7 I, 38 \mathrm{I}-40 \mathrm{I}$.

Crurea I. : Prohemistomum appendiculatum, eine neue Holostomiden-Art aus Hunden- und Katzen-darm, dessen Infektionsquelle inden Süsswasserfischen zu suchen ist. $Z$. InfektKr., I9I6, I7, 309-328.

Dennis E. A., Penner L. R. : Mesostephanus yedeae sp. n. (Trematoda : Cyathocotylidae), its life history and descriptions of the developmental stages. Occ. Pap. Univ. Conn. (Biol.), I97 I, 2, 5-I 5 .

Dubors G. : Monographie des Strigeida (Trematoda). Mém. Soc. neuchatel. Sci. nat., r938, 6, I-535.

Dubors G. : Nouvelle clé de détermination des groupes systématiques et des genres de Strigeida Poche (Trematoda). Rev. suisse Zool., r951, s8, 639-691.

Dubors G. : Les Strigeida (Trematoda) de Californie de la Collection June Mahon. Bull. Soc. neuchatel. Sci. nat., 1958, 81, 69-78.

Dubors G. : Notes Helminthologiques II : Diplostomatidae Poirier et Cyathocotylidae Poche (Trematoda). Rev. suisse Zool., 1969a, 76, 3-21.

Dubors G. : Sur trois Cyathocotylidés (Trematoda : Strigeata). Ann. Parasitol. Hum. Comp., $\mathrm{I} 969 \mathrm{~b}, 44,547-554$.

Dubors G. : Les Strigeata (Trematoda) de la Collection A. Lutz. Mem. Inst. Oswaldo Cruz, 1970, 68, I69-I96.

Dubois G. : Comparaison des genres Gogatea Lutz, 1935 et Neogogatea Chandler et Rausch, I947 (Strigeata : Cyathocotyloidea : Prohemistomidae). Bull. Soc. neuchatel. Sci. nat., I975a,198, 39-4I.

Dubors G. : Sur quelques Mesostephanus Lntz, I935 (Strigeata : Cyathocotyloidea). Ann. Parasitol. Hum. Comp., 1975b, so, 447-459.

Dubois G. : Du statut de quelques Strigeata La Rue, I926 (Trematoda). V. Bull. Soc. neuchâtel. Sci. nat., 1977, 100, 35-44.

Dubors G. : Notes Helminthologiques IV : Strigeidae Railliet, Diplostomidae Poirier, Proterodiplostomidae Dubois et Cyathocotylidae Poche (Trematoda). Rev. suisse Zool., 1978, 85, 607-61 5 .

Dubois G., ANGel L. M. : Strigeata (Trematoda) of Australian birds and mammals from the helminthological collection of the University of Adelaide. Trans. R. Soc. S. Aust., 1972, 96, I97-2I 5 .

Dubois G., Angel L. M. : Mesostephanus neophocae n. sp. (Strigeata : Prohemistomidae), parasite d'une Otarie d'Australie, Neophoca cinerea (Péron et Lesueur). Bull. Soc. neuchâtel. Sci. nat., 1976, 99, 29-32.

Dubois G., PEARson J.-C. : Les Strigeida (Trematoda) d'Égypte (Collection William H. Wells). Ann. Parasitol. Hum. Comp., 1963, 38, 77-91.

Dubors G., Pearson J.-C. : Quelques Strigeida (Trematoda) d'Australie. Bull. Soc. neuchatel. Sci. nat., $1965,88,77-99$.

Dubors G., Pearson J.-C. : Ibid., II. Bull. Soc. neuchâtel. Sci. nat., I967, 90, 185-204.

Fischthal. J. H., Kuntz R. E. : Some Trematodes of Amphibians and Reptiles from Taiwan. Proc. helminth. Soc. Wash., $\mathrm{x} 975,42, \mathrm{I}-\mathrm{I} 3$.

Hoffman G. L., Dunbar C. E. : Studies on Neogogatea kentuckiensis (Cable, 1935) n. comb. (Trematoda : Strigeoidea : Cyathocotylidae). J. Parasitol., 1963, 49, 737-744.

Hutton R. F., Sogandares-Bernal F. : Studies on helminth parasites from the coast of Florida. II. Digenetic trematodes from shore birds of the west coast of Florida. I. Bull. Mar. Sci. Gulf and Caribbean, I960 $a, 10,40-54$.

Hutton R. F., Sogandares-Bernal F. : Preliminary notes on the life-history of Mesostephanus appendiculatoides (Price, 1934) Lutz, 1935. Bull. Mar. Sci. Gulf and Caribbean, 1960b, 10, $234-236$.

Lutz A. : Observaçoes e consideraçoes sobre Cyathocotylineas e Prohemistomineas. Mem. Inst. Osw. Cruz, Rio de J., I935, 30, I57-168 (trad. allem., 169-182). 
Malczewski A. : Helminth parasites of bred foxes and minks in Poland. Acta Parasit. polon., $1962,10,231-260$.

Malczewski A. : Mesostephanus alopicis sp. n. Bull. Acad. polon. Sci., Cl. 2, 1964, 12, 91-94.

Martin W. E. : Life cycle of Mesostephanus appendiculatus (Ciurea, 1916) Lutz, 1935 (Trematoda : Cyathocotylidae). Pacif. Sci., 1961, 15, 278-281.

Mehra H. R. : On certain new and already known distomes of the family Cyathocotylidae Poche from northern India with a discussion on the classification of the family. Proc. Indian Sci. Congr., 1943, 30, 70-7I (Abstract).

Merra H. R. : Studies on the family Cyathocotylidae Poche. Part 2. A contribution to our knowledge of the subfamily Prohemistominae Lutz, 1935, with a discussion on the classification of the family. Proc. Nat. Acad. Sci., India, 1947, I7, I-52.

Mikhail J. W., Faнmy M. A. M. : Two new species of Trematodes from Insectivores. Zool. Anz., I $968,181,42 I-426$.

MYeR D. G. : On the life history of Mesostephanus kentuckiensis (Cable, 1935) n. comb. (Trematoda : Cyathocotylidae). J. Parasitol., 1960, 46, 819-832.

Oshmarin P. G., Mamaev Yu. L., Lebedev B. I. : [Helminths of animals from South-east Asia.] Nauka, Moskva, 1970, pp. 5-126.

Perrov A. M. : Nouveau trématode de l'intestin du chat, Mesostephanus skworzowi nov. sp. (Strigeata) ]. Trudi Vsesoy. Inst. Gelmint. Acad. K. I. Skrjabin, 1950, 4, 8I-82.

Prendel A. R. : Ein Beitrag zum Studium der Helminthenfauna der Hunde in der U.d.S.S.R. (Südliche Ukraine). Zool. Anz., 1930, 89, 323-326.

Price E. W. : The occurrence of Prohemistomum appendiculatum in the United States. J. Parasitol., $1928, I S, 68$.

Price E. W.: New trematode parasites of birds. Smithson. Misc. Coll., I934, 9I, I-6.

RICHARD J. : Trematodes d'oiseaux de Madagascar. Note IV. Strigéides et Cyathocotylides. Bull. Mus. Hist. nat., Paris, 1964, 36, 506-522.

Sudarikov V. E., in SkrJabin K. I. : Trématodes des animaux et de l'homme. Éléments de trématodologie]. Acad. Sci. U.R.S.S., 1961, 19, 267-471.

Travassos L. : Nouvelle espèce de Prohemistomum. C. R. Soc. Biol. (Sect. brés.), 1924, 91, 835-838.

Tubangui M. A. : Two new intestinal trematodes from the dog in China. Proc. U. S. Nat. Mus., $1922,60, \mathrm{I}-\mathrm{I} 2$.

Tubangui M. A., Masilungan V. A. : Trematode parasites of Philippine vertebrates. IX. Flukes from the domestic fowl and other birds. Philipp. J. Sci., I94I, 75, I3 I-I42.

Vidyarthi R. D. : Some new members of the family Cyathocotylidae Poche, I925 from Indian birds. Indian J. Helminthol., I948, I, 23-40.

Yamaguti S. : Studies on the Helminth Fauna of Japan. Part 25. Trematodes of Birds, IV. Jap. Journ. Zool., 1939, 8, 129-210. 